\title{
THE COMPLICATIONS OF DIFFERENTIAL DIAGNOSTICS OF HODGKIN LYMPHOMA
}

\author{
Iryna Kriachok \\ Department of Chemotherapy of Hemoblastosis with Adjuvant Methods of Treatment \\ National Cancer Institute \\ 33/43 Lomonosova str., Kyiv, Ukraine, 03022 \\ irina.krychok@unci.org.ua \\ Katerina Ulianchenko \\ Department of Chemotherapy of Hemoblastosis with Adjuvant Methods of Treatment \\ National Cancer Institute \\ 33/43 Lomonosova str., Kyiv, Ukraine, 03022 \\ kate.ulianchenko@gmail.com
}

\begin{abstract}
In the article are presented the questions of classification, pathogenesis and clinical diagnostics of Hodgkin Lymphoma with soft-tissue paravetebral component. The difficulties of early diagnostics, expressed at the expanse by oncological process localization, not typical for this disease, are shown on clinical case.

The symptoms, conditioned by the compression of organs by tumor masses depend on pathological process localization: intrahepatic and extrahepatic obstruction of bile duct is manifested by jaundice; obstruction of lymphatic ducts in groin zone or in zone of small pelvic - by legs edema; at tracheobronchial compression is observed the pant and hoarse breathing; pulmonary abscesses or caverns can take place as a result of infiltration of pulmonary parenchyma that can stimulate lobar consolidation or bronchopneumonia; paraplegia can be observed because of epidural invasion with spine cord compression; compression of sympathetic cervical recurrent guttural nerve by increased lymph nodes can cause Horner's syndrome and laryngoparalysis; neuralgias can be the result of nerve-root incarceration.

Differential diagnostics can take a long time that lowers the effectiveness of therapy and decreases chances for recovery because of special treatment delay. The setting of correct diagnosis and choice of adequate therapy raises the chances for recovery, even at spead stage of tumor process that is attained at Hodgkin lymphoma in most cases.
\end{abstract}

Keywords: Hodgkin lymphoma, schwannoma, peripheral T-cellular lymphoma, diagnostics, classification, risk groups, treatment.

\section{Introduction}

In most cases the diagnostics of Hodgkin Lymphoma is not difficult but in some cases is observed non-typical manifestation of disease, connected with localization of tumor masses near spine cord and nerve trunks with correspondent neurological symptomatology.

\section{Aim}

To consider the difficulties of diagnostic search, features of clinical course of Hodgkin lymphoma with soft-tissue paravetebral component on the example of clinical case.

\section{Description of clinical case}

The man, 27 years old was admitted to the department of oncohematology of National cancer institute (NCI) with complaints on pain between shoulder-blades, feeling of numbness in legs, expressed weakness, convulsions, impossibility to move independently. The anamnesis of disease: felt ill since the beginning of 2015, when started to observe the numbness, weakness in right leg, disturbance of perceptibility in legs. He addressed to neurologist domiciliary. He underwent magnetic-resonance tomography of thoracic spine: MR picture of extra- and intradural volume mass at the level of Th1-Th5 vertebra on the left, Th10-Th12 vertebra on the right and Th12-L1 vertebra on the left; stenosis of vertebral canal at the level of Th1-Th4 with the signs of myelopathy. The signs of injury were in the body of Th9, Th12 vertebra. The biopsy of mass was carried out at neurolog- 
ical department of Kiev hospital: hemilaminectomy of Th1-Th4 on the left, partial elimination of tumor (intravertebral component was totally eliminated) at the level of Th1-Th4 vertebra. Decompression of spine cord. Histologic conclusion: PHC № 7067-7113/15 - schwannoma.

Schwannoma (neurinoma, schwannoglioma, lemmoblastoma, perineural fibroplastoma) - tumor, growing from Schwann cells that form myelin nerve coat. The term was offered by Verokai and widely spread.

Schwannomas are benign tumors, are observed in any age, more often in women. Most often localization is auditory nerve. They can occur on any nerves of human organism [1].

Macroscopically schwannoma looks as a dense, limited node of oval or odd shape. The node surface is uneven, bumpy. Schwannoma is covered with connective capsule. The tissue of tumor on the cut is faintly grey, with part of yellow, rusty tint at the expanse of fatty deposition or brown color (the evidence of old hemorrhages). The color of tissue can change depending on conditions of tumor blood supply: at venous congestion it acquires cyanotic tint. In tumor tissue often occur cysts of different sizes, filled with brown liquid. Cystic degeneration can be detected in whole tumor or its part. The spread fields of fibrosis are often observed.

The diverse transformations, appeared as the result of dystrophic processes, inherent to schwannomas, caused the separation of different histologic types: epithelioid - with dense placement of cells and weak expressiveness of fibrous tissue; angimatous - with mass of cavernous cavities; xanthomatous - with significant crowd of xanthome cells.

Schwannomas (neurinomas) usually don't infiltrate the surrounding tissues but cause their intensive compression [2]. Malignization is rather seldom. It is attended with change of morphological presentation that acquires similarity with so-called neurogenic sarcomas. Clinical presentation depends on schwannoma localization. The symptoms of irritation and compression of spine cord develop at schwannomas of roots of spine cord. At schwannomas of peripheral nerves the clinical presentation consists of symptoms of irritation and prolapsed of functions of correspondent nerves. At typical forms of neurinomas the clinical course is slow, benign. Magnetic-resonance tomography is a method of choice at diagnostics. The treatment of disease is mainly surgical; the radial therapy is used at large sizes of tumor or when surgical intervention is technically impossible. The prognosis of disease is mainly favorable; after adequate treatment disease is completely eliminated without consequences for patient.

After pathohistologic diagnosis as schwannoma patient was sent for further treatment and examination to the department of oncohematology of CNI (Cancer national institute). Objectively: general status is relatively satisfactory (ECOG 1). Neurological status: is conscious, oriented in space, palpebral fissure $\mathrm{D}=\mathrm{S}$, pupils $\mathrm{D}=\mathrm{S}$, photoreaction is a little lowered. Tendon reflexes are high, on the right higher than at the left, knee and Achilles - with polykinetic response. The deep low pyramidal paraparesis of low extremities, decrease of muscular force in legs is $0-1$ point, hypesthesia at the level Th5-Th6, stagger in Romberg position, finger-nasal test is executed with miss-hit from the right. Babinsky symptom is negative from two sides, meningeal signs are absent.

The general blood analysis - insignificant leucocytosis, ESR acceleration, in biochemical blood analysis were observed hypoproteinuria, insignificant increase of AcT and AlT. In myelogram from iliac bone: blast cells $-2,25$; lymphocytes $-4,25$; promyelocytes $-0,25$; myelocytes $-17,25$; y. $-5,25 ; \mathrm{st} / \mathrm{k}-10,25 ; \mathrm{sig} / \mathrm{k}-17,7$ erythrocyte appendage $-40,75$; plasmatic cells -2 . The function of megacaryocytic appendage is preserved. In biopsy material from iliac bone is the normal presentation of marrow.

According to CT data of neck, breast organs, small pelvis organs: thrombosis of internal jugular vein. Conglomerate of increased lymph nodes is at the level of aorta bifurcation $(1 / \mathrm{n})$ $45 \times 39 \mathrm{~mm}$, paraaortic $1 / \mathrm{n} 23 \times 22 \mathrm{~mm}$. Iliac $1 / \mathrm{n}$ on the right $29 \times 31 \mathrm{~mm}$, on the left $18 \times 53 \mathrm{~mm}$. Obturative $1 / \mathrm{n}$ on the right $23 \times 55 \mathrm{~mm}$, on the left $27 \times 57 \mathrm{~mm}$. Groin $1 / \mathrm{n}$ on the right $32 \times 27 \mathrm{~mm}$, on the left $30 \times 29 \mathrm{~mm}$.

In pathohistologic CNI department the biopsy material was revised: pathohistologic conclusion № 7067-7113/15, immune-histochemical study № 2307/15: CD20-; CD3+; CD4 +; CD8-; Ki-67 to $22 \%$; Granzyme B-, CD 30-. Conclusion: peripheral T-cellular lymphoma (NOS). 
Unclassified peripheral T-cellular lymphoma - tumors, generated from mature (post-thymic) T-lymphocytes and NK-cells. This rare group of diseases is characterized with pathobiological heterogenity and is near $15 \%$ of all lymphomas. This category is the diagnosis of exclusion that includes all T-cellular lymphomas, not related to other subvariants, according to WHO classification. The timorous cells are characterized with clonal rebuilding of T-cellular receptor, often CD3 and CD30-positive. Specific genetic and immune- phenotypic features are absent [3].

In connection with disagreement of diagnoses, according to the conclusions of CNI and Kiev hospital, conference made a decision about revision of biopsy material in one more laboratory. The second histologic and immune-histochemical study was carried out: CD20+; CD3-; CD15+; CD30+; CD5-; OCT2 weakly+; BOB 1-; Ki-67 + mainly in most timorous cells. Conclusion: histologic creation of tumor cells and immune phenotype correspond to classic Hodgkin lymphoma, mixed-cellular variant.

In connection with received three different results it was recommended to revise preparation in one more laboratory. According to the results of repeated studies, the tumor cells expressed CD20+; CD3-; CD15+; CD30+; CD45-. Conclusion: histologic structure of tumor cells and immune phenotype correspond to classic Hodgkin lymphoma, variant of nodular sclerosis.

Hodgkin lymphoma (HL) - malignant disease of lymphoid tissue, which morphological substrate is a presence of huge cells of Reed-Beresovsky-Stenberg at microscopic study of injured lymphatic nodes. This variant of lymphoma is $11 \%$ of all lymphomas. Morbidity is 2,9 for 100000 people. Men have it more often than women (1,4:1). Hodgkin lymphoma can appear at any age, but there are two peaks of morbidity: 20-29 years old and at the age older than 55 year. As opposite to other lymphatic malignant mass, Hodgkin lymphoma is not associated with typical genetic injuries [4].

There are two main forms of this disease: classic Hodgkin lymphoma that is $95 \%$ of all cases and nodular type of lymphoid prevalence of Hodgkin disease (Hodgkin lymphoma with lymphoid prevalence, HLLP) $-5 \%$ of cases. These forms differ by histologic presentation and clinical course (Table 1). Nodular Hodgkin lymphoma with lymphoid prevalence is characterized with more favorable prognosis. This form is more often occurs in men 30-50 years old.

Table 1

Determination of HL risk groups in correspondence with EORTC/LYSA and GHSG classifications

\begin{tabular}{ccc}
\hline Stages & Classification according to EORTC/LYSA & Classification according to GHSG \\
\hline Early stages & CS I-II (epiphrenic injury) without risk factors & CS I-II Without risk factors \\
Intermediate stages & CS I-II (epiphernic injury) $\geq 1$ risk factor & CS IIA $\geq 1$ risk factor; \\
Spread stages & CS III-IV & CS IIB with risk factors C/D, without A/B factors A/B, CS III/IV \\
& (A) big mass in mediastinum & (A) big mass in mediastinum \\
(B) age $\geq 50$ years old & (B) presence of extranodal injury \\
(C) increased ESR & (C) increased ESR
\end{tabular}

Note: CS - clinic stage, EORTC - European Organisation for Research and Treatment of Cancer, ESR - erythrocyte sediment rate, GHSG - German Hodgkin Study Group, LYSA - Lymphoma Study Association

The classic Hodgkin lymphoma is divided in four subtypes by histologic signs: nodular sclerosis or sclerosing lymphoma ( $70 \%$ of cases): most spread in USA, more often in women and young patients. Mixed-cellular (20\% of cases): most spread among patients with HIV. Rich in lymphocytes (3-5 \% of cases). Lymphoid exhaustion (2\% of cases). Lymphoid exhaustion was only recently more spread subtype but since diagnostic methods in oncology were improved, the number of cases of this variant of disease decreased.

The symptoms, conditioned by compression of organs by timorous masses depend on pathologic process localization: intra-hepatic and extra-hepatic obstruction of bile duct is manifested by jaundice; obstruction of lymphatic ducts in groin zone or in zone of small pelvic - by legs edema; at 
tracheobronchial compression is observed the pant and hoarse breathing; pulmonary abscesses or caverns can take place as a result of infiltration of pulmonary parenchyma that can stimulate lobar consolidation or bronchopneumonia; paraplegia can be observed because of epidural invasion with spine cord compression; compression of sympathetic cervical recurrent guttural nerve by increased lymph nodes can cause Horner's syndrome and laryngoparalysis; neuralgias can be the result of nerve-root incarceration.

The diagnosis Hodgkin Lymphoma (HL) is set according to the criteria of World Health Organization (WHO) on the base of the study of operational material, excisional or incisional biopsy of lymphatic node under condition of sufficient quantity of diagnostic material. For histologic presentation of classis HL is typical the reactive microenvironment and big timorous cells of Hodgkin or Reed-Sternberg (HRS-cells), whereas for the variant of nodular HL with lymphoid prevalence is necessary to find LP-cells (big cells with multiblade kernels, reminding of popcorn) [4, 5]. HRScells are positive at coloration with antibodies to CD30 and CD15, sometimes CD20-positive (part of timorous cells) and negative at antibodies use to CD45. LP-cells are characterized with monomorphic expression of CD20 and CD45, but don't express CD15 and CD30 [6].

The full recovery after use of standard polychemotherapy (PCT) courses can be attained in more than $80 \%$ of patients (ESMO book).

For the choice of therapy practice it is necessary to determine the risk group of patient before the beginning of treatment. The risk group of patient depends on number of factors of unfavorable prognosis. These factors include: ESR increase $>50 \mathrm{~mm} /$ hour at absence of B-symptoms and $>30 \mathrm{~mm} /$ hours at B-symptoms; big mass in mediastinum (mass with size as more than one third of maximal horizontal diameter of chest), B-symptoms (increase of body temperature, night sweats, unexplained loss of body weight $>10 \%$ during 6 months), presence of extranodal injury and more than 3 involved $1 / n$ zones.

The treatment of HL spread stages.

The standard of treatment at spread stages is chemotherapy. The radial therapy (RT) is used in some patients after chemotherapy [7].

Patients at $\leq 60$ years old can receive from six to eight cycles according to ABVD scheme, with further local RT on residual nidi of timorous injury with size more than $1,5 \mathrm{~cm}$ or six cycles of BEACOPP escalated, with further local RT on PET-positive residual timorous nidi with size more than $2,5 \mathrm{~cm}$.

The treatment results according to BEACOPP escalated scheme are possibly better comparing with ones according to ABVD. The treatment according to BEACOPP escalated scheme is attended with more toxicity and needs the associated therapy $[8,9]$.

BEACOPP escalated must not be used in patients $>60$ years because of high mortality, connected with treatment [10].

ABVD is a standard of treatment for elder patients that multicomponent chemotherapy can be applied in. According to the data of several studies [14, 15], intermediate (PET/CT study after 1-2 courses of PCT has a great prognostic importance.

After repeated revision of all consulting conclusions of 3 pathohistologic laboratories and biopsy material there was set diagnosis as Hodgkin lymphoma of IV A stage with injury of Th1-Th5 vertebra on the left, Th10-Th12 vertebra on the right and Th12-L1 vertebra on the left, conglomerate of increased lymphatic nodes, paaraortic lymphatic nodes, ilioinguinal lymphatic nodes, obtrutive lymphatic nodes at the level of aorta bifurcation. The status after hemilaminectomy operation (09.11.15) of Th1-Th4 on the left, partial elimination of tumor (intravertebral component is totally eliminated) at the level of Th1-Th4 vertebra. Decompression of spine cord. Thrombosis of internal jugular vein.

In connection with aggressive debut of disease and spreading of process, patient underwent the specific therapy according to intensive chemotherapy scheme BEACOPP-esc (6 courses). Rated doses: Cyclophosphamide $1250 \mathrm{mg} / \mathrm{m}^{2}-2600 \mathrm{mg}$, Doxorubicine $35 \mathrm{mg} / \mathrm{m}^{2}-70 \mathrm{mg}$, Etoposide $200 \mathrm{mg} / \mathrm{m}^{2}-$ $400 \mathrm{mg}$ (1-3 days, totally $1200 \mathrm{mg}$ ), Bleomycin $10 \mathrm{mg} / \mathrm{m}^{2}-21 \mathrm{mg}$, Vincristine $1.4 \mathrm{mg} / \mathrm{g}^{2}-2 \mathrm{mg}$, Prednisolone $40 \mathrm{mg} / \mathrm{m}^{2}-80 \mathrm{mg}$ (1-14 days totally $1120 \mathrm{mg}$ ), procarbazine $-100 \mathrm{mg} / \mathrm{m}^{2} 200 \mathrm{mg}$ (1-7 days 
totally $1400 \mathrm{mg}$ ). Associated therapy: ondansetron $8 \mathrm{mg}$ № 5, heptral $400 \mathrm{mg}$ № 14, uromitexan - 400 mg № 6, allopurinol $600 \mathrm{mg} / \mathrm{day}, \mathrm{mg} / \mathrm{day}$, lansoprazole $30 \mathrm{mg} /$ day.

The complications developed after therapy: leucopenia of 4 degree to $0,02 \mathrm{~g} / 1$. In connection with it patient received clonal-stimulating factors, antibacterial therapy, antifungal therapy. For assessment of intermediate response patient underwent PET-CT, after 4 courses of BEACOPP-esc. According to PET-CT data, in spine soft tissues at Th1-Th5 level was visualized RPP with intensity $\mathrm{SUV}=6.1$. The signs of residual mass are Deauville criteria score 3. (PET-negative) that testifies to the absence of timorous activity.

\section{Results}

After the end of study PET-CT was also carried out. According to the data of PET-CT, the absence of biologically active masses was observed. The assessment of response was carried out according to Deauville criteria. The full metabolic response to the treatment was attained.

\section{Conclusions}

The full recovery at Hodgkin lymphoma is possible in $80-90 \%$ of patients [4], including at injury of spine cord.

It is very seldom, when clinical presentation of Hodgkin lymphoma conditions the significant complications in early diagnostics that is principally important for timely beginning of specific therapy.

The injury of lymphatic nodes in combination with extramedular intradural volume neoplasm usually occurs at non-Hodgkin lymphomas of extranodal type or peripheral T-cellular lymphomas, NOS, and is no typical for HL that complicates and delays the terms of diagnosis setting as it was presented in considered clinical case. The term from the moment of first histologic diagnosis to the setting of final one and beginning of the therapy was 6 months.

Neurological symptomatology and localization of process could correspond to the schwannoma diagnosis, but because of spreading of process, biopsy material was sent to another laboratory for revision. The disagreement of diagnoses in different laboratories always indicates the complicated diagnostic case. In this situation it is expedient to address to conference and set the correct diagnosis together.

The setting of correct diagnosis before the beginning of therapy is a guarantee of treatment effectiveness as it was demonstrated on the example of patient. Despite the severe status and large spreading of process, the adequately prescribed treatment allowed attaining the full response to therapy.

\section{References}

[1] Yamakami, I., Ito, S., Higuchi, Y. (2014). Retrosigmoid removal of small acoustic neuroma: curative tumor removal with preservation of function. Journal of Neurosurgery, 121 (3), 554-563. doi: 10.3171/2014.6.jns132471

[2] Kohno, M., Shinogami, M., Yoneyama, H., Nagata, O., Sora, S., Sato, H. (2014). Prognosis of Tinnitus After Acoustic Neuroma Surgery - Surgical Management of Postoperative Tinnitus. World Neurosurgery, 81 (2), 357-367. doi: 10.1016/j.wneu.2012.09.008

[3] Willemze, R., Hodak, E., Zinzani, P. L., Specht, L., Ladetto, M. (2013). Primary cutaneous lymphomas: ESMO Clinical Practice Guidelines for diagnosis, treatment and follow-up. Annals of Oncology, 24, vi149-vi154. doi: 10.1093/annonc/mdt242

[4] Eichenauer, D. A., Engert, A., Andre, M., Federico, M., Illidge, T., Hutchings, M. et. al. (2014). Hodgkin's lymphoma: ESMO Clinical Practice Guidelines for diagnosis, treatment and follow-up. Annals of Oncology, 25, iii70-iii75. doi: 10.1093/annonc/mdu181

[5] Farrell, K., Jarrett, R. F. (2011). The molecular pathogenesis of Hodgkin lymphoma. Histopathology, 58 (1), 15-25. doi: 10.1111/j.1365-2559.2010.03705.x

[6] Mahoney, K. M., Atkins, M. B. (2014). Prognostic and predictive markers for the new immunotherapies. Oncology (Williston Park), 28, 39-48. 
[7] Visco, C., Nadali, G., Vassilakopoulos, T. P., Bonfante, V., Viviani, S., Gianni, A. M. et. al. (2006). Very high levels of soluble CD30 recognize the patients with classical Hodgkin's lymphoma retaining a very poor prognosis. European Journal of Haematology, 77 (5), 387-394. doi: 10.1111/j.1600-0609.2006.00725.x

[8] Diehl, V., Fuchs, M. (2007). Will BEACOPP be the standard for high risk Hodgkin lymphoma patients in advanced stages? Transfusion and Apheresis Science, 37 (1), 37-41. doi: 10.1016/j.transci.2007.04.004

[9] Bosetti, C., Levi, F., Ferlay, J., Lucchini, F., Negri, E., La Vecchia, C. (2009). The recent decline in mortality from Hodgkin lymphomas in central and eastern Europe. Annals of Oncology, 20 (4), 767-774. doi: 10.1093/annonc/mdn673

[10] Cerhan, J. R., Slager, S. L. (2015). Familial predisposition and genetic risk factors for lymphoma. Blood, 126 (20), 2265-2273. doi: 10.1182/blood-2015-04-537498

[11] Johnson, P. W. et. al. (2015). See International Prognostic Score (IPS) (HODG-A). ddAdapted from the RATHL study. Hematol. Oncol., 33, 102.

[12] Kostakoglu, L., Evens, A. M. (2014). FDG-PET Imaging for Hodgkin lymphoma: current use and future applications. Clin. Adv. Hematol. Oncol., 12 (1), 20-35.

[13] Sokolowska-Wojdylo, M., Olek-Hrab, K., Ruckemann-Dziurdzinska, K. (2015). Primary cutaneous lymphomas: diagnosis and treatment. Advances in Dermatology and Allergology, 5, 368-383. doi: 10.5114/pdia.2015.54749

[14] Hutchings, M., Kostacoglu, L., Zaucha, J. M. et. al. (2011). Early determination of treatment sensivity in Hodgkin Lymphoma: FDG-PET after one cycle of therapy has a higher negative predictive value than after two cycles of chemotherapy. Ann. of Onc., 22 (4), 162.

[15] Gallamini, A., Tarella, C., Patti, C. et. al. (2011). Multicentral clinical study with early treatment intensification in high-risk Hodgkin lymphoma patients, with a positive FDG-PET scan after two ABVD courses - GITIL HD0607 study. Ann. of Onc., 22 (4), 163. 